\title{
MIOPATÍA POR CUERPOS DE INCLUSIÓN. CASO CLÍNICO Y REVISIÓN DE LA LITERATURA
}

\author{
Claudia M. Guzmán V., MD..$^{1 *}$, María F. Cubides, MD. ${ }^{2}$, Elsa ReYes S. MD. ${ }^{3}$, \\ John Londoño, MD. ${ }^{3}$, Pedro Santos, MD. ${ }^{3}$, Wilson Bautista, MD. ${ }^{1}$, Rosa Salazar, Md. ${ }^{2}$, \\ Paola Varela, MD. ${ }^{2}$ Y Rafael Valle O. MD. ${ }^{3}$ \\ ${ }^{1}$ Residente del Programa de Reumatología, Universidad Militar Nueva Granada, Bogotá; ${ }^{2}$ Residente del \\ Programa de Reumatología, Universidad de la Sabana, Bogota; ${ }^{3}$ Reumatólogo, Servicio de Reumatología \\ Hospital Militar Central, Bogotá, Colombia, Universidad Militar Nueva Granada y Universidad de la Sabana.
}

\section{Resumen}

La miositis por cuerpos de inclusión (MCI) es una patología poco común que se presenta en individuos mayores de 50 años y con mayor frecuencia en hombres; sin embargo, los síntomas pueden empezar 20 años antes de su diagnóstico. Hasta un $30 \%$ de los casos de miopatías inflamatorias pueden ser de $\mathrm{MCI}$ y su prevalencia varía en los diferentes países y grupos étnicos. Clínicamente se manifiesta como debilidad lentamente progresiva proximal y distal. En este artículo se presenta un caso de una paciente de 78 años con antecedentes previos de diabetes mellitus tipo 2 , hipertensión arterial, e hipotiroidismo, con cuadro de 18 meses de evolución consistente en debilidad de miembros inferiores, incapacidad para subir y bajar escaleras, limitación en la marcha, acompañado de disestesias. Se realizó la medición de la creatin quinasa (CK) y su valor inicial fue 7820, la electromiografía y neuroconducciones mostraron polineuropatia axonal motora en las cuatro extremidades y miopatía inflamatoria. Se trató con glucorticoides a dosis de $1 \mathrm{mg} / \mathrm{kg}$ y después de seis meses de tratamiento no se observó mejoría. Se decide tomar biopsia muscular que mostró miopatía por cuerpos de inclusión, lo que explicó la falla terapéutica inicial. Este caso es interesante desde el punto de vista clínico, ya que no es frecuente la elevación de la CK en los niveles que presentó la paciente y por las múltiples comorbilidades asociadas.

Palabras clave: miositis, miopatía, cuerpos de inclusión.

\section{INCLUSION BODY MYOPATHY. CASE REPORT AND LITERATURE REVIEW}

\begin{abstract}
Inclusion body myositis (IBM) is an infrequent disease in people over 50 years of age, affecting more frequently males; however the symptoms may begin 20 years before its diagnosis. Up to $30 \%$ of all inflammatory myopathies can be IBM and its prevalence varies among different countries and ethnical groups. IBM manifest clinically by slowly progressive proximal and distal weakness. In this article a report of case is presented: A female patient 78 years old with previous history of type 2 diabetes mellitus, hypertension and hypothyroidism; with 18 months history of weakness in lower limbs, inability to climb stairs, limited to walk, associated with dysesthesias. The initial CK was $7820 \mathrm{mg} / \mathrm{dl}$, the electromyography and nerve conductions reported a motor axonal polyneuropathy of four limbs and inflammatory myopathy. She was treated with steroids at doses of $1 \mathrm{mg} / \mathrm{kg}$ without improvement, after 6 months of treatment. It was decided to take a muscle biopsy that showed an inclusion body myopathy, explaining
\end{abstract}

* Correspondencia: Claudia Guzmán cm_guzman75@hotmail.com. Dirección postal: Tr.3 \#49-00. Servicio de Reumatología, Hospital Militar Central, Bogotá Colombia.

Recibido: Abril 5 de 2010 Aceptado: Mayo 26 de 2010. 
the initial treatment failure. This case is interesting from a clinical point of view, since it is not frequent elevated CK levels as it was presented by the patient and for the numerous associated co-morbidities.

Keywords: myositis, myopathy, inclusion bodies

\author{
MIOPATIA POR CORPOS DE INCLUSÃO. \\ RELATO DE CASO E REVISÃO DA LITERATURA
}

\begin{abstract}
Resumo
A miosite por corpo de inclusão (MCI) é uma doença rara que apresenta-se em indivíduos acima de 50 anos, atingindo $30 \%$ das miopatias inflamatória; orém os sintomas podem começar 20 anos antes. Sua prevalência varia entre países e grupos étnicos. Clínicamente manifesta-se como uma fraqueza lentamente progressiva proximal e distal mais frecuentemente nos homens. Nós relatamos um caso de uma paciente de 78 anos com antecedentes de diabetes mellitus tipo 2, hipertensão e hipotireoidismo. Com quadro clínico de 18 meses de evolução consistindo em fraqueza nos membros inferiores, incapacidade de subir escadas, a limitação para a marcha, acompanhados de disestesias. Foi feito a prova de CK dando um valor inicial de 7820 e com eletromiografia e neurocondução que informaram polineuropatia axonal motora de quatro membros e miopatia inflamatória. Foi tratado com corticóides em doses de $1 \mathrm{mg} / \mathrm{kg}$ sem melhora após 6 meses de tratamento, razão pela qual foi decidido fazer uma biópsia do músculo que mostrou miopatía do corpo de inclusão, explicando a falha do tratamento inicial. Este caso é interessante do ponto de vista clínico, uma vez que não é frequente a elevação da $\mathrm{CK}$ nos niveis apresentados pela paciente e pelas múltiplas comorbidades associadas.
\end{abstract}

Palavras-chave: miosite, doenças musculares, corpos de inclusão

\section{Introducción}

La miopatía por cuerpos de inclusión (MCI) es una patología que se presenta en individuos mayores de 50 años y con mayor frecuencia en hombres y junto con la polimiositis, la dermatomiositis y la miopatía necrotizante conforman las miopatías idiopáticas inflamatorias mediadas en forma inmune (1-4); sin embargo, son clínica e histológicamente diferentes $(3,5)$. El primer caso de $\mathrm{MCI}$ fue descrito por Chou en 1967 en un hombre de 66 años con un diagnóstico de polimiositis crónica, pero en la biopsia muscular se pudo observar las inclusiones citoplasmáticas y fibras musculares con vacuolas (6); el término MCI fue introducido en 1971 por Yunis y Samaha (7) y luego en 1991 Mendell y col. utilizaron la tinción de Rojo Congo e identifican por primera vez la presencia de amiloide en las fibras musculares (8). Debido a que el tratamiento de la $\mathrm{MCI}$ es distinto al de las otras miopatías, su diagnóstico diferencial es muy importante (9). En Colombia son pocos los casos reportados, solo encontramos cinco en el Valle del Cauca en pacientes menores de 40 años (10).

\section{Reporte del caso}

Paciente femenina de 78 años de edad, enfermera pensionada procedente de Bogotá, con antecedente de diabetes mellitus tipo 2, hipertensión arterial, hipotiroidismo, asma en tratamiento desde hace cinco años. Consulta por cuadro de 18 meses de evolución consistente en debilidad muscular de predominio en miembros inferiores, asociado a dificultad para subir $y$ bajar escaleras, limitación en la marcha y disestesias. Inicialmente es valorada por el servicio de neurología que la reporta como paciente con pérdida importante de la fuerza, debilidad muscular para cambios de posición sentada y bipedestación. Marcha ligeramente parética, reflejos osteotendinosos normales, con niveles de CPK de 7819, por lo cual se decide hospitalizarla para estudio de miopatía.

Durante la hospitalización se realizaron: 1- estudios inmunológicos tales como la medición de anticuerpos antinucleares (ANAS) reportándose un valor de 1/320 con patrón moteado fino y de anticuerpos extractables del núcleo (ENAS) que fueron reportados 
como negativos; 2- estudios para neoplasias como la tomografía axial computada toracoabdominal que mostró colelitiasis y miomatosis uterina calcificada; 3- neuroconducción y electromiografía de las cuatro extremidades en donde se muestra polineuropatía axonal motora en las cuatro extremidades y presencia de miopatía inflamatoria. Después de descartar procesos neoplásicos, infecciosos y problemas por medicamentos, se diagnostica una miopatía inflamatoria y se inicia el tratamiento con prednisona $1 \mathrm{mg} / \mathrm{kg} / \mathrm{día}$, observándose una mejoría parcial de la sintomatología.

Seis meses después se observa que persiste la debilidad en miembros inferiores hasta el punto de incapacitarla para caminar. Se remite al servicio de reumatología, donde se decide ampliar los estudios inmunológicos, se miden los niveles de anticuerpos anti- músculo liso, anti-mitocondriales, anti-JO y de crioglobulinas; se hacen estudios para vasculitis (ANCAS), se ordena una electroforesis de proteínas y todos los resultados son negativos. Otros exámenes realizados y sus resultados fueron: ANAS 1/160 con patrón moteado fino, ENAS negativo, anticuerpos anti-DNA negativo. Teniendo en cuenta la edad de la paciente, la falta de respuesta al tratamiento y las coomorbilidades asociadas, se decide solicitar biopsia muscular (tablas 1 y 2 ).

En el informe de la biopsia muscular, con la coloración de eosina se observan vacuolas dentro de las fibras musculares y proceso inflamatorio intersticial y se concluye que el diagnóstico es miopatía inflamatoria por cuerpos de inclusión (Imagen 1). La biopsia de nervio sural fue informada con vasculitis leve.

\section{Miopatía por cuerpos de inclusión}

La MCI es uno de los desórdenes musculares adquiridos y su patogénesis es desconocida; se considera que hay una interacción compleja entre la edad, la genética y los factores ambientales. Las características patológicas son una triada única: cambios inflamatorios con invasión de linfocitos $\mathrm{CD} 8+$ que se expresan en las fibras musculares, con inclusiones citoplasmáticas e intranucleares con contenido amiloide, que interactúan con desórdenes miodegenerativos $(11,12)$, los cuales se han visto asociados a trastornos inmunes e infecciosos y que son disparadores de la MCI (tabla 3). Desde el punto de vista de la epidemiología se han descrito diferencias en su incidencia en los diferentes grupos étnicos, siendo baja en los coreanos, afroamericanos y mestizo mesoamericano (13), media en la población
TABLA 1. Resultados de los exámenes de laboratorio.

\begin{tabular}{lcc}
\hline Examen & $\begin{array}{c}\text { Valores al } \\
\text { inicio }\end{array}$ & $\begin{array}{c}\text { Valores a los seis } \\
\text { meses }\end{array}$ \\
\hline CK & 7819 & 7285 \\
LDH & 2140 & 1770 \\
TGP & 394 & 534 \\
TGO & 257 & 314 \\
Hemoglobina & 16,7 & 16,5 \\
Nitrógeno ureico & 22 & 14,9 \\
Creatinina & 0,6 & 0,4 \\
TSH & 11,50 & 1,80 \\
\hline
\end{tabular}

Valores en mg /dl. CK (Creatin kinasa), LDH (Deshidrogenasa láctica), TGO (Transaminasa glutámico pirúvica), TGO (Transaminasa glutámico oxalacética)

TABLA 2. Resultados de pruebas inmunológicas

\begin{tabular}{lc}
\hline \multicolumn{1}{c}{ Examen inmunológico } & Valor \\
\hline ANAS & $1 / 160$ patrón moteado fino \\
ENAS & Negativo \\
Anti-Jo 1 & Negativo \\
Anti Mitocondrial & Negativo \\
Factor reumatoide & Negativo \\
Electroforesis de proteínas & Normal \\
Crioglobulinas & Negativo \\
\hline
\end{tabular}

ANAS (anticuerpos antinucleares), ENAS (anticuerpos antinucleares extraíbles del núcleo).

del este y sureste del Mediterráneo y alta en el norte de Europa y en la población blanca de Norteamérica y Australia.

Las manifestaciones clínicas se presentan usualmente después de los 50 años, pero el diagnóstico se realiza cinco a ocho años después del inicio de los síntomas que son insidiosos, con debilidad que se desarrolla en forma lenta y progresiva, generalmente inician de la parte proximal a la distal y afecta más a los hombres que a las mujeres (tabla 4) (31-33). En algunos casos se ve más comprometido el sensorio y la disfagia puede llegar a presentarse en el $40 \%$ de los casos, 

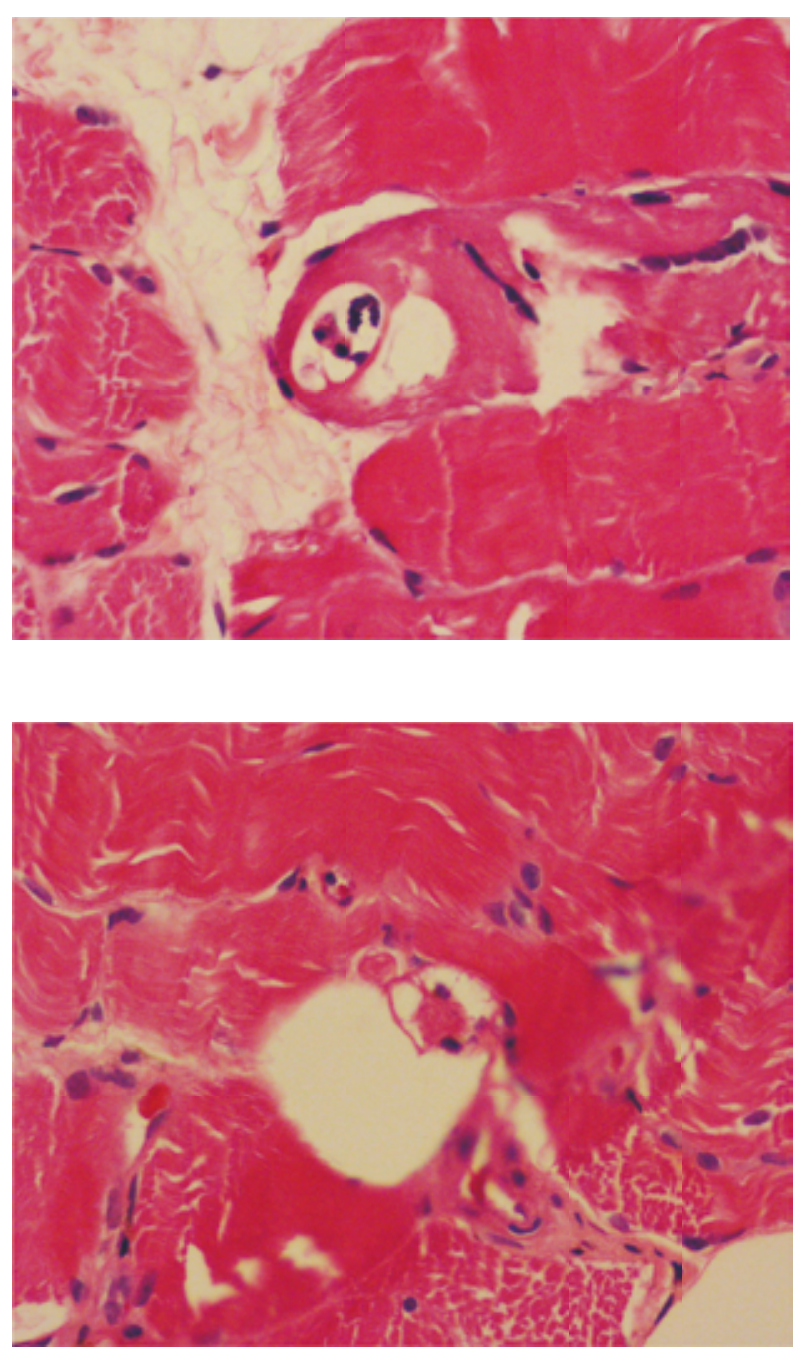

FIGURA 1. Fotografía de la biopsia de músculo con microscopia de luz (cortesía Dr. Fernando Velandia, Servicio de patología Hospital Militar Central).

requiriendo miotomía cricofaríngea la cual es beneficiosa $(34,35)$. La afección del sensorio se examina clínicamente y con estudios electrofisiológicos y de neuroconducción, que evidencian la neuropatía axonal sensorial $e$ incremento de la actividad polifásica motora $(36,37)$. Los estudios de laboratorios pueden mostrar elevación de la CK sérica la cual puede estar normal o elevada hasta diez veces (38,39); los anticuerpos antinucleares pueden estar presentes en un $20 \%$ de los casos, pero usualmente están ausentes y un $20 \%$ de los pacientes pueden tener una gamapatía monoclonal indeterminada. La resonancia magnética puede mostrar afectación muscular focal, atrofia y definir los patrones de pérdida de unidades motoras después
TABLA 3. Desórdenes inmunológicos e infecciosos asociados a la miositis por cuerpos de inclusión.

\begin{tabular}{l}
\hline \multicolumn{1}{c}{ Inmunológicos } \\
\hline Inmunodeficiencias comunes variables (14) \\
Púrpura Trombocitopénica Idiópatica $(15,16)$ \\
Síndrome de Sjögren (17,18) \\
Dermatomiositis (19) \\
Otras enfermedades del tejido conectivo: \\
Lupus eritematoso, esclerodermia, artritis reumatoidea (20,21) \\
Paraproteinemias (22) \\
Síndrome antisintetasa y otras miositis asociadas (23) \\
\hline Infecciosas \\
\hline Virus de Inmunodeficiencia Humana (24) \\
Virus de la hepatitis C (25,26) \\
Toxoplasmosis (28) \\
\hline \multicolumn{1}{c}{ Otros desórdenes (raros) } \\
\hline Sarcoidosis (27) \\
Miositis macrofágica (29) \\
Post poliomelitis (30) \\
\hline
\end{tabular}

TABLA 4. Signos predictores de miopatía por cuerpos de inclusión

Debilidad en flexores de las manos, extensores de la muñeca

Atrofia y debilidad del cuadriceps

Asimetría de antebrazos

Disfagia

Neuropatía periférica

Presencia de ANAS en el $20 \%$ de los pacientes

Creatincinasa sérica normal o elevada diez veces lo normal

Electromiografía

Biopsia muscular

del traumatismo de nervios periféricos, por cual es importante para el diagnóstico clínico y seguimiento de respuesta al tratamiento (40-43).

Para realizar el diagnóstico diferencial es importante tener en cuenta la clínica, los paraclínicos y la histopatología confirmada, para definir si es MIC o si estamos ante otra patología (Tabla 5). La expectativa de vida no se altera en la $\mathrm{MCI}$, pero desafortunadamente es una enfermedad lentamente progresiva (44-46). 
TABLA 5. Diagnóstico diferencial de la miopatía por cuerpos de inclusión

\begin{tabular}{l} 
Miopatias Inflamatorias Idiopáticas \\
\hline Polimiositis \\
Dermatomiositis \\
Miositis asociada a otras enfermedes del colágeno y vasculitis \\
Miositis asociada a neoplasia maligna
\end{tabular}

Otras formas de miopatia inflamatoria

Miositis asociada a eosinofilia

Miositis localizada o focal

\section{Miopatía Causada por Infección}

Adenovirus, citomegalovirus, coxsakie, hepatitis $\mathrm{B}$, virus de inmunodeficiencia

Humana, cándida, criptopcoco, estafilococo, estreptococo, tuberculosis, otros.

Miopatia causada por fármacos y toxinas

Cloroquina, colchicina, D-penicilamina, glucocorticoides, etc.

En general MIC no responde a los inmunosupresores o inmunomoduladores y algunos pacientes terminan dependientes luego de diez a quince años de iniciada la enfermedad. Se han realizado varios estudios para medir la eficacia de los corticoides, pero la respuesta no ha sido tan positiva como se esperaba; los resultados mostraron una disminución de los niveles séricos de $\mathrm{CK}$, pero sin cambios en la fuerza muscular ni en la histopatología $(47,48)$. Los estudios realizados con medicamentos citotóxicos como el metotrexate mostraron los mismos resultados (49). En los estudios en donde se utilizó la inmunoglobulina humana, se observó una diferencia significativa en la mejoría de los síntomas neuromusculares, pero no en la fuerza muscular, ni una diferencia en los niveles de $\mathrm{CK}$, pero se considera una buena alternativa con buen perfil de seguridad (50-52). Otros trabajos realizados en los que se utilizaron globulina anti-timocito o terapias basadas en citoquinas (interferón $\alpha$, interferón $\beta$ 1a, etanercept, alentuzumab), los resultados no tuvieron impacto; además se concluye que se requiere un nuevo estudio que incluya un mayor número de pacientes y más tiempo de observación para definir su eficacia y seguridad (53-56).

Los avances en la biotecnología han promovido nuevos productos como los anticuerpos monoclonales o fusión de proteínas para la inmunoterapia, que manipulan el sistema inmune por inhibición selectiva o depleción de células $\mathrm{B}$ o células $\mathrm{T}$; se pueden citar el daclizumab, antagonista del receptor de IL-2; alentuzumab, anticuerpo monoclonal humanizado contra señalización de células $\mathrm{T}$; fingomilod, agente anti-migración de la célula T; eculizumab, anticuerpo monoclonal agonista del factor $\mathrm{C} 5$ del complemento y natalizumab, agente antimigración de células $\mathrm{T}$ o B (57-62). Todos estos agentes se han utilizado con éxito en esclerosis múltiple, pero en las miopatías inflamatorias sólo se han hecho estudios piloto. Se recomienda que las terapias farmacológicas se deben asociar a otras terapias, que dependen de cada caso, como la miotomía cricofaríngea, la inyección con toxina botulínica en el esfínter esofágico, la terapia física y ocupacional que son eficaces y seguras en el reacondicionamiento aeróbico del paciente, el uso de ortesis es beneficioso y son bien toleradas por los pacientes y la trasferencia de tendones en algunos pacientes que restaura la función, sobre todo en los extensores de los dedos (63-69). Otras terapias empíricas como la oxalondrona, un andrógeno sintético, ha dado buen resultado sobre la fuerza muscular; la coenzima Q10, la carnitina, los agentes antioxidantes y el clerbuterol mejoran los síntomas de los pacientes. Se espera que estos nuevos productos sean mejorados para un adecuado aprovechamiento en la terapéutica de estos desórdenes, que de alguna manera siguen siendo un reto para el médico en el manejo de estos pacientes (70).

\section{Conclusión}

La miopatía por cuerpos de inclusión es una patología que se debe reconocer tempranamente, ya que su diagnóstico y tratamiento difieren de otras miopatías más frecuentes. Requiere de una integración clínica, paraclínica e histopatológica específica, como se describe en el caso informado. Existen varios tratamientos incluso en fase II, pero ninguno ha sido determinante para el manejo definitivo de esta entidad.

\section{Referencias}

1. Amato A A, Gronseth G S, Jackson C E et al. Inclusion Body Myositis: Clinical and Pathological Boundaries. Annals of Neurology. 1996;40:581-6.

2. Lotz B P, Engel A G, Nishino H et al. Inclusion Body Myositis: Observation in 40 Patients. Brain. 1989;112:727-47.

3. Amato A A, Barohn R J. Inclusion Body Myositis: Old and New Concepts. Journal of Neurology, Neurosurgery and Psychiatry. 2009;80;1186-1193.

4. Griggs R C, Askanas V, DiMauro S et al. Inclusion Body Myositis and Myopathies. Annals of Neurology. 1995;38:705-13

5. Dalakas M C. Advance Online Publication. Nature Reviews Rheumatology. 2 February 2010. 
7. Chou S M. Myxovirus-like Structures in a Case of Human Chronic Polymyositis. Science. 1967;158:1453-55.

8. Yunis E J, Samaha F J. Inclusion Body Myositis. Laboratory Investigation. 1971;25:240-48.

9. Mendell J R, Sahenk Z, Gales T, Paul L. Amyloid Filaments in Inclusion Body Myositis: Novel Findings Provide Insight into Nature of Filaments. Archives of Neurology. 1991;48:122934.

10. Barohn R J, Amato A A. Inclusion Body Myositis. Current Treatment Options in Neurology. 2000;2:7-12.

11. Vallejo G, Medina L F, Garcia L. Acta Médica Colombiana. 2007;32(2).

12. Askanas V, Engel WK. Inclusion Body Myositis: a Myodegenerative Conformational Disorder Associated with A $\beta$ Protein Misfolding, and Proteasome Inhibition. Neurology. 2006;66:39-48.

13. Dalakas M C. Sporadic Inclusion Body Myositis- Diagnosis, Pathogenesis, and Therapeutic Strategies. Nature Clinical Practice. 2006;2:437-45.

14. Griggs R C, Askansas V, DiMauro S et al. Inclusion Body Myositis and Myopathies. Annals of Neurolology. 1995;38:705-13.

15. Dalakas M C, Illa I. Common Variable Immunodeficiency and Inclusion Body Myositis: a Distinct Myopathy Mediated by Naturalkiller Cells. Annals of Neurology. 1995;37:806-10.

16. Riggs J E, Schochet S S Jr, Gutmann L, McComas C F, Rogers $\mathrm{J} S$ 2nd. Inclusion Body Myositis and Chronic Immune, Thrombocytopenia. Archives of Neurology. 1984;41:93-95.

17. Williams S F, Mincey B A, Calamia K T. Inclusion Body Myositis Associated with Celiac Sprue and Idiopathic Thrombocytopenic Purpura. Southern Medical Journal. 2003;96:721-23.

18. Kanellopoulos P, Baltoyiannis C, Tzioufas A G. Primary Sjögren's Syndrome Associated with Inclusion Body Myositis. Rheumatology. 2002;41:440-44.

19. Danon M J, Perurena O H, Ronan S, Manaligod J R. Inclusion Body Myositis Associated with Systemic Sarcoidosis. Canadian Journal of Neurological Sciences. 1986;13:334-36.

20. McCoy A L, Bubb M R, Plotz P H, Davis J C. Inclusion Body Myositis Long after Dermatomyositis: a Report of Two Cases. Clinical and Experimental Rheumatology. 1999;17:235-39.

21. Limaye V, Scott G, Kwiatek R, Pile K. Inclusion Body Myositis Associated with Systemic Lupus Erythematosus. Australian and New Zealand Journal of Medicine. 2000;30:275-76.

22. Kim S, Genth E, Krieg T, Hunzelmann N. PM-Scl Antibody Positive Systemic Sclerosis Associated with Inclusion-Body Myositis. Rheumatology. 2005;64:499-502.

23. Dalakas M C, Illa I, Gallardo E, Juarez C. Inclusion Body Myositis and Paraproteinemia: Incidence and Immunopathologic Correlations. Annals of Neurology. 1997;41:100-04.

24. Koff man B M, Rugiero M, Dalakas M C. Autoimmune Diseases and Autoantibodies Associated with Sporadic Inclusion Body Myositis. Muscle and Nerve. 1998;21:115-17.

25. Cupler E J, Leon-Monzon M, Miller J, Semino-Mora C, Anderson TL, Dalakas M C. Inclusion Body Myositis in HIV-1 and HTLV-1 Infected Patients. Brain. 1996;119:1887-93.

26. Yakushiji Y, Satoh J, Yukitake $\mathrm{M}$ et al. Interferon Beta-Responsive Inclusion Body Myositis in a Hepatitis C Virus Carrier. Neurology. 2004;63:587-88.

27. Alexander J A, Huebner C J. Hepatitis C and Inclusion Body Myositis. The Amercian Journal of Gastroenterology. 1996;91:1845-47.

28. Bouillot S, Coquet M, Ferrer X, Lagueny A, Leroy J P, Vital C.Inclusion Body Myositis Associated with Sacroidosis: A Report of 3 Cases. Annals of Pathology. 2001;21:334-36.
29. Mastaglia F L, Phillips BA. Idiopathic Infl ammatory Myopathies: Epidemiology, Classifi cation, and Diagnostic Criteria. Rheumatic Diseaseas Clinics of North America. 2002;28:72341.

30. Cherin P, Menard D, Mouton P et al. Macrophagic Myofasciitis Associated with Inclusion Body Myositis: A Report of Three Cases. Neuromuscular Disorders. 2001;11:452-57.

31. Parissis D, Karkavelas G, Taskos N, Milonas I. Inclusion Body Myositis in a Patient with a Presumed Diagnosis of Post-Polio Syndrome. Journal of Neurology. 2003;250:619-21.

32. Lotz B P, Engel A G, Nishino $\mathrm{H}$ et al. Inclusion Body Myositis: Observation in 40 Patients. Brain. 1989;112:727-47.

33. Mhiri C, Gherardi R. Inclusion Body Myositis in French Patients. A Clinicopathological Evaluation. Neuropathology and Applied Neurobiology. 1990;16:333-44.

34. Badrising U A, Maat-Schieman M L, Van Houwelingen J C et al. Inclusion Body Myositis: Clinical Features and Clinical Course of the Disease in 64 Patients. Journal of Neurology. 2005;252:1448-54.

35. Verma A, Bradley W G, Adesina A M et al. Inclusion Body Myositis with Cricopharyngeus Muscle Involvement and Severe Dysphagia. Muscle and Nerve. 1991;14:470-3.

36. Darrow D H, Hoffman H T, Barnes GJ et al. Management of Dysphagia in Inclusion Body Myositis. Archives of Otolaryngology- Head and Neck Surgery. 1992;118:313-17.

37. Eisen A, Berry K, Gibson G. Inclusion Body Myositis (IBM): Myopathy or Neuropathy? Neurology. 1983;33:1109-14.

38. Joy J L, Oh S J, Baysal A I. Electrophysiological Spectrum of Inclusion Body Myositis. Muscle Nerve. 1990;13:949-51.

39. Lotz B P, Engel A G, Nishino H. Inclusion Body Myositis: Observation in 40 Patients. Brain. 1989;112:727-47.

40. Griggs R C, Askansas V, DiMauro S et al. Inclusion Body Myositis and Myopathies. Annals of Neurology. 1995;38:705-13.

41. Koffman B M, Rugiero M, Dalakas MC. Immune-Mediated Conditions and Antibodies Associated with Sporadic Inclusion Body Myositis. Muscle Nerve. 1998;21:115-17.

42. Love L A, Leff R L, Fraser D. A New Approach to the Classification of Idiopathic Inflammatory Myopathy: Myositis-Specific Autoantibodies Define Useful Homogeneous Patient Groups. Medicine (Baltimore). 1991;70:360-74.

43. Fraser D D, Frank J A, Dalakas M. Magnetic Resonance Imaging in the Idiopathic Inflammatory Myopathies. The Journal of Rheumatology. 1991;18:1693-700.

44. Sekul E A, Chow C, Dalakas M C. Magnetic Resonance Imaging of the Forearm as a Diagnostic Aid in Patients with Sporadic Inclusion Body Myositis. Neurology. 1993;48:863-6.

45. Lacomis D. Neurophatic Disorders and Muscular Dystrophy. In Worthmann R L. Disease of Skeletal Muscle. Philadelphia. Lippincott, Williams and Milkins. 2000:197-220.

46. Rudnicki S A, Dalmau J. Paraneoplastic Syndromes of the Spinal Cord, Nerve, and Muscle Nerve. Muscle Nerve. 2000;23:1800-18.

47. Griggs R C, Askansas V, DiMauro S. Inclusion Body Myositis and Myopathies. Annals of Neurology. 1995;38:705-13.

48. Barohn R J, Amato A A, Sahenk Z, Kissel J T, Mendell J R. Inclusion Body Myositis: Explanation for Poor Response to Immunosuppressive Therapy. Neurology. 1995;45:1302-04.

49. Askanas V, McFerrin J, Alvarez R B, Baque S, Engel W K. $\beta A P P$ Gene Transfer into Cultured Human Muscle Induces Inclusion Body Myositis Aspects. NeuroReport. 1997; 8: 2155-58.

50. Badrising U A, Maat-Schieman M L, Ferrari M D. Comparison of Weakness Progression in Inclusion Body Myositis during 
Treatment with Methotrexate or Placebo. Annals of Neurology. 2002;51:369-72.

51. Leff R L, Miller F W, Hicks J, Fraser D D, Plotz P H. The Treatmentof Inclusion Body Myositis: A Retrospective Review and a Randomized, Prospective Trial of Immunosuppressive Therapy. Medicine. 1993;72:225-35.

52. Soueidan SA, Dalakas MC. Treatment of Inclusion-Body Myositis with High-Dose Intravenous Immunoglobulin. Neurology. 1993;4:876-9.

53. Amato A A, Barohn R J, Jackson C E, Pappert E J, Sahenk Z, Kissel J T. Inclusion Body Myositis: Treatment with Intravenous Immunoglobulin. Neurology. 1994;444:1516-18.

54. Lindberg C, Trysberg E, Tarkowski A, Oldfors A. Anti-T Lymphocyte Globulin Treatment in Inclusion Body Myositis. Neurology. 2003;61:260-62.

55. Group TMS. Randomized Pilot trial of Beta INF1a (Avonex) in Patients with Inclusion Body Myositis. Neurology. 2001;57:1566-70

56. Group TMS. Randomized Pilot Trial of High-Dose INF-1a in Patients with Inclusion Body Myositis. Neurology. 2004; 63: 718-20.

57. Barohn R J, Herbelin L, Kissel J T. Pilot Trial of Etanercept in the Treatment of Inclusion Body Myositis. Neurology. 2006;66:123-24.

58. Rose J W et al. Daclizumab Phase II Trial in Relapsing and Remitting Multiple Sclerosis: MRI and Clinical Results. Neurology. 2007;69:785-789.

59. Dalakas $\mathrm{M} C$ et al. Effect of Alemtuzumab (CAMPATH 1-H) in Patients with Inclusion Body Myositis. Brain. 2009;132:15361544.

60. O'Connor P et al. Oral Fingolimod (FTY720) in Multiple Sclerosis: Two-year Results of a Phase II Extension Study. Neurology. 2009;72:73-79.

61. Kissel J T, Mendell J R, Rammohan K W. Microvascular Deposition of Complement Membrane Attack Complex in Dermatomyositis. New England Journal of Medicine. 1986;314:329 334.
62. Dalakas M C. B Cells as Therapeutic Targets in Autoimmune Neurological Disorders. Nature Clinical Practice Neurology. 2008;4:557-567.

63. Basta M, Dalakas M C. High-Dose Intravenous Immunoglobulin Exerts, its Beneficial Effect in Patients with Dermatomyositis by Blocking Endomysial Deposition of Activated Complement Fragments. The Journal of Clinical Investigation. 1994;94 :1729-35.

64. Cherin P, Pelletier S, Teixeira A. Intravenous Immunoglobulin for Dysphagia of Inclusion Body Myositis. Neurology. 2002;58:326-7.

65. Darrow D H, Hoff man H T, Barnes G J, Wiley C A. Management of Dysphagia in Inclusion Body Myositis. Archives of Otolaryngology- Head and Neck Surgery. 1992;118:313-17.

66. Liu L W, Tarnopolsky M, Armstrong D. Injection of Botulin Toxic A to the Upper Esophageal Sphincter for Oropharyngeal Dysphagia in two Patients with Inclusion Body Myositis. The Canadian Journal of Gastroenterology. 2004;18:397-99.

67. Arnardottir S, Alexanderson H, Lundberg I E, Borg K. Sporadic Inclusion Body Myositis: Pilot Study on the Eff ects of a Home Exercise Program on Muscle Function, Histopathology and Infl ammatory Reaction. Journal of Rehabilitation Medicine. 2003;35:31-35.

68. Heikkila S, Vittanen J V, Kautianen H. Rehabilitation in Myositis. Physiotherapy. 2001;87:301-09.

69. Spector S A, Lemmer J T, Koff man B M. Safety and Efficacy of Strength Training in Patients with Sporadic Inclusion Body Myositis. Muscle Nerve. 1997;20:1242-48.

70. Waclawik A J, Rao V K. Effective Treatment of Severe Finger Flexion Weakness in Inclusion Body Myositis Using Tendon Transfers. Journal of Clinical Neuromuscular Disease. 2002;4:31-32.

71. Rutkove S B, Parker R A, Nardin R A. A Pilot Randomized Trial of Oxandrolone in Inclusion Body Myositis. Neurology. 2002;58:1081-87. 